Ann. Biol. anim. Bioch. Biophys., I962, 2 (2), I29-142

\title{
EFFETS D'UNE CARENCE SÉVÈRE DE RIBOFLAVINE SUR LA POULE PONDEUSE : ORDRE DE PRIORITÉ ENTRE LES TISSUS MATERNELS ET L'CEUF
}

\author{
J.C. BLUM \\ avec la collaboration technique de I. MaCAIRE \\ Centre de Recherches sur la Nutrition du C.N.R. S., Bellevue (Seine-et-Oise.)
}

\section{SOMMAIRE}

Privée de riboflavine, la poule pondeuse transfère cette vitamine dans l'œuf aux dépens de ses propres tissus. Elle peut ainsi sacrifier plus de la moitié de son capital hépatique en riboflavine pour assurer la ponte. Le poids du foie est proportionnellement plus diminué que sa teneur en vitamine $B_{2}$. 'Toute la riboflavine hépatique n'est pas également disponible. Une certaine proportion constitue une réserve immédiatement mobilisable pour l'ovogenèse. Lorsque cette réserve est épuisée, le foie devient très avare de sa riboflavine et la teneur des oufs baisse considérablement, puis la ponte s'arrête. Le besoin vitaminique de ponte n'est donc pas prioritaire : la poule ne lui sacrifie ses réserves tissulaires que dans la mesure où ce transfert ne nuit pas à son propre équilibre nutritionnel. Cet ordre de priorité ressort également du fait que lors de réplétion en vitamine $\mathrm{B}_{2}$ les réserves sont d'abord reconstituées, l'activité ovarienne ne reprenant que plus tard.

Toute la riboflavine du blanc d'œuf provient des réserves accumulées dans le magnum avant passage du vitellus. Chez la poule pondeuse non carencée, ces réserves sont localisées selon un gradient de concentration et leur importance diminue quand on passe de la partie antérieure à la partie postérieure du magnum. En régime carencé en riboflavine, le magnum ne se recharge pas normalelent en vitamine $\mathrm{B}_{2}$ et on n'observe plus de gradient de répartition entre les parties antérieure et postérieure.

A la suite d'autres auteurs, nous avons montré qu'un régime très pauvre en riboflavine entraîne une diminution progressive de la teneur des œufs en cette vitamine avant de provoquer un arrêt de ponte (BLUM, CALET, JACQUOT, I96I). Nous nous sommes demandés si la carence affectait davantage l'organisme de la poule pondeuse que les produits de la ponte. Autrement dit, dans quelle mesure la riboflavine tissulaire peut-elle être transférée à l'œuf lors d'une déficience d'apport alimentaire ? 


\section{MATÉRIEL ET MÉTHODES}

Pour répondre à la question que nous venons de poser, nous avons cherché à réaliser un régime pratiquement dépourvu de riboflavine, mais contenant par ailleurs tous les nutriments indispensables. Dans les produits naturels, la vitamine $B_{2}$ s'est avérée très résistante bien qu'on la considère comme un facteur photosensible. Même dans un milieu liquide comme le lait et à un $\mathrm{pH}$ légèrement alcalin, la riboflavine n'est que très lentement détruite par irradiation aux rayons UV. Nous avons donc eu recours à un régime semi-synthétique comprenant : $20 \mathrm{p}$. 10o de gluten (soit I $5 \mathrm{p}$. I00 de protides), o,4 p. Ioo de DL lysine, 6o p. Ioo d'amidon, 5 p. Ioo d'huile d'arachide, 3 p. Ioo de cellulose, I 1,6 p. Ioo de sels et vitamines (selon les normes de Fisher et JoHnson, 1956). Ce régime carencé (régime $\mathrm{C}$ ) ne contenait que des traces de riboflavine $(0,4 \mathrm{mg} / \mathrm{kg}$ ). A notre connaissance un régime aussi pauvre en vitamine $B_{2}$ n'avait jamais été administré à des poules pondeuses. Des témoins recevaient le même régime semi-synthétique supplémenté en riboflavine à raison de ro $\mathrm{mg}$ par $\mathrm{kg}$ d'aliment (régime S).

A partir du I 3 mars, 40 Poules habituées à pondre en batterie reçoivent le régime $\mathrm{S}$ supplémenté en riboflavine. Cette prépériode se poursuit jusqu'au 6 avril. Ce jour-là, toutes les poules sont pesées et 4 d'entre elles sacrifiées. Le reste des animaux est alors divisé en 2 lots :

- le lot $\mathrm{S}$ (I8 poules) continue à recevoir le régime supplémenté en vitamine $\mathrm{B}_{\mathbf{2}}$;

- le lot C (I8 poules) est placé au régime carencé en riboflavine.

Tous les ro jours, quatre poules de chacun des lots expérimentaux sont pesées et sacrifiées en vue du dosage de riboflavine dans le foie, la grappe ovarienne et l'oviducte. Dès avant et pendant la prépériode ( 13 mars- 6 avril) et tout au long de la période expérimentale ( 7 avril-1 5 mai), l'intensité de ponte est contrôlée et les œufs sont recueillis et pesés. La riboflavine est dosée séparément dans l'albumen et le vitellus en groupant pour chaque poule les œufs appartenant à la même série. Nous avons utilisé la méthode microbiologique de SNELL et STRONG (1939).

Le Io mai, trois poules du lot carencé (régime C) reçoivent chacune une dose unique de to $\mathrm{mg}$ de riboflavine tandis que trois poules du lot témoin (régime $\mathrm{S}$ ) sont mises au régime $\mathrm{C}$. Ces animaux sont sacrifiés le 15 mai en mème temps que les trois poules restant dans le lot $\mathrm{S}$. Aucune poule du lot $\mathrm{C}$ ne survivait par suite de morts accidentelles.

Le schéma général de l'expérience a donc été le suivant :

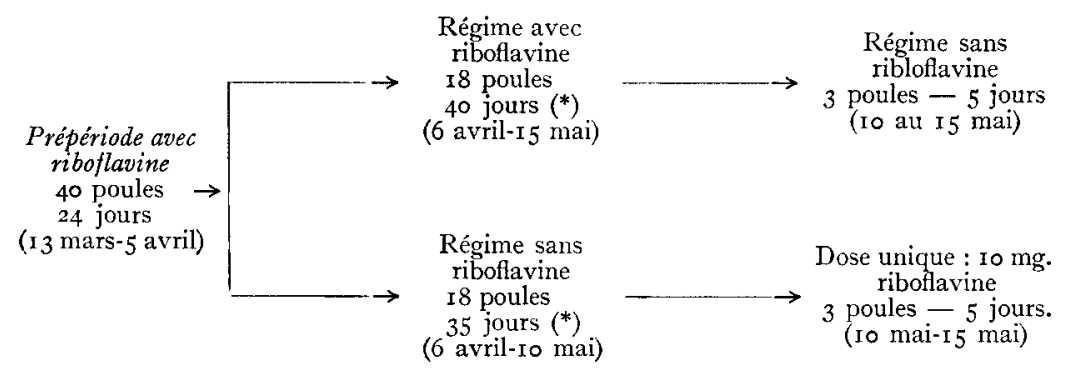

\section{RÉSULTATS EXPÉRIMENTAUX ET DISCUSSION}

\section{I. - RÉPARTITION DE LA RIBOFLAVINE ENTRE LE FOIE, IAA GRAPPE OVARIENNE ET L'OEUF}

\section{Variations de l'intensité de ponte}

I, a variation de l'intensité de ponte (nombre moyen d'cufs pondus chaque jour par cent poules) est rapportée pour chaque lot dans le tableau I.

(*) Il s'agit de la durée maximum ne tenant pas compte des sacrifices en cours de période. 
TABLEAU I

Variation de l'intensité de ponte

\begin{tabular}{|c|c|c|c|c|c|c|c|}
\hline $\begin{array}{l}7-14 \\
\text { mars }\end{array}$ & $\begin{array}{l}15-25 \\
\text { mars }\end{array}$ & $\begin{aligned} 26 & \text { mars } \\
5 & \text { avril }\end{aligned}$ & $\begin{array}{l}6-16 \\
\text { avril }\end{array}$ & $\begin{array}{r}17-26 \\
\text { avril }\end{array}$ & $\begin{array}{l}27-30 \\
\text { avril }\end{array}$ & $\begin{array}{l}1-7 \\
\text { mai }\end{array}$ & $\begin{array}{l}8-16 \\
\text { mai }\end{array}$ \\
\hline \multirow{3}{*}{72} & \multirow{3}{*}{73} & \multicolumn{4}{|c|}{ Lot ténoin (régime $\mathrm{S}: 10 \mathrm{mg}$ riboflavine $/ \mathrm{kg}$ ) } & \multirow{3}{*}{55} & \multirow{3}{*}{59} \\
\hline & & 46 & 30 & 48 & 58 & & \\
\hline & & \multicolumn{4}{|c|}{ Lot carencé (régime $\mathrm{C}: 0,{ }^{\prime} \neq \mathrm{mg}$ riboflavine/lig) } & & \\
\hline 77 & 74 & 43 & 25 & 40 & 10 & 0 & 0 \\
\hline \multicolumn{2}{|c|}{$\begin{array}{l}\text { Mise en prépériode } \\
\text { des } 2 \text { lots (régime } S \text { ) }\end{array}$} & \multicolumn{2}{|c|}{$\begin{array}{c}\text { Mise en carence } \\
\text { du lot } C \text {. }\end{array}$} & & & & \\
\hline
\end{tabular}

Au cours de la prépériode une baisse sensible de l'intensité de ponte intervient une dizaine de jours après la mise au régime expérimental $\mathrm{S}$.

Cette diminution s'accentue par la suite chez les témoins maintenus au régime $\mathrm{S}$; puis après 4 semaines environ, la ponte reprend, se stabilisant toutefois à un niveau plus bas qu'à l'origine. Bien qu'enrichi en riboflavine, ce régime $S$ était imparfait: valeur biologique des protéines médiocre, valeur énergétique trop élevée et rapport énergie-protéines défectueux. On peut supposer que ces défauts sont à l'origine du ralentissement de ponte qui s'accompagnait d'une diminution progressive du poids de l'œuf atteignant son maximum (- Io p. roo) entre le 6 et le I 6 avril. Puis les poules semblent avoir retrouvé un nouvel équilibre métabolique : la ponte reprend, se stabilise et le poids de l'œuf ne varie plus.

Chez les poules carencées placées au régime $\mathrm{C}$, la reprise de ponte est freinée avant un arrêt brutal. Ici toute stabilisation est impossible du fait de la déficience d'apport en riboflavine.

\section{Analyse du foie}

Dans le tableau 2 figurent le poids du foie, la teneur en riboflavine par gramme de foie frais et le contenu hépatique total en cette vitamine.

TABLEAU 2

Caractéristiques moyennes (poids et richesse en riboflaine) du foie

\begin{tabular}{|c|c|c|c|c|c|c|}
\hline \multirow{2}{*}{$\begin{array}{l}\text { Date du } \\
\text { sacrifice }\end{array}$} & \multicolumn{3}{|c|}{ Lot témoin (régime S) } & \multicolumn{3}{|c|}{ Lot carencé (régime C) } \\
\hline & Poids (g) & $\begin{array}{l}\text { Contenu total } \\
\text { en riboflavine } \\
(\mu \mathrm{g})\end{array}$ & $\begin{array}{l}\text { Teneur en } \\
\text { riboflavine } \\
(\mu \mathrm{g} / \mathrm{g} \text { frais })\end{array}$ & Poids (g) & $\begin{array}{l}\text { Contenu total } \\
\text { en riboflaxine } \\
(\mu g)\end{array}$ & $\begin{array}{l}\text { Teneur en } \\
\text { riboflavine } \\
\text { ( } \mu g / g \text { frais) }\end{array}$ \\
\hline 6 avril ..... & 36,3 & 533 & 15,0 & & & \\
\hline 17 avril ..... & 62,7 & $6+1$ & 10,2 & $50,,^{\prime}$ & 445 & 8,9 \\
\hline 27 avril .. & 61,2 & 784 & 12,9 & 36,6 & 336 & 9,1 \\
\hline 8 mai.. & 68,6 & 777 & 11,2 & 31,6 & 243 & 7,7 \\
\hline \multirow[t]{2}{*}{15 mai.. } & 70,3 & 727 & 10,9 & - & $一$ & - \\
\hline & \multicolumn{3}{|c|}{ Régime C à partir du 10 mai } & \multicolumn{3}{|c|}{$\begin{array}{l}\text { Administration per os d'une dose } \\
\text { unique de riboflavine, le } 10 \mathrm{mai} \\
\text { (10 mg par poule) }\end{array}$} \\
\hline 15 mai....... & 41,5 & 430 & 10,3 & 68,1 & 643 & 9,4 \\
\hline
\end{tabular}


Les animaux sacrifiés le 6 avril se différencient nettement dans le lot témoin $\mathrm{S}$ : le poids du foie est faible, ce qui est à rattacher à une intensité de ponte diminuée.

La carence en vitamine $B_{2}$ a un effet très net sur le poids du foie qui ne fait que diminuer à mesure que l'état carentiel se poursuit. Au contraire dans le lot témoin, le poids du foie s'élève avec le temps, suivant l'augmentation de l'indice de ponte.

La carence vitaminique diminue la concentration de riboflavine dans le foie. Si on prend les chiffres đu 8 mai, on relève un écart de plus de 30 p. Ioo entre les teneurs par gramme de foie frais du lot témoin et du lot carencé.

Cependant, l'influence de l'avitaminose étant plus manifeste pour le poids du foie que pour sa concentration en riboflavine, il s'ensuit que la diminution de la riboflavine totale hépatique est surtout imputable à une réduction de la masse de l'organe.

D'une manière générale, les teneurs en riboflavine du foie sont assez faibles même chez les animaux au régime S. Le fait peut être imputé à la valeur biologique médiocre des protides du régime. On sait, en effet, que les réserves hépatiques de vitamine $B_{2}$ dépendent, pour une large part, de la quantité et de la qualité des protides alimentaires (ADRIAN, LuNVEN et TERROINE, Ig6I).

Dix milligrammes de riboflavine administrés per os à la poule carencée suffisent pour restaurer le poids du foie et reconstituer son capital vitaminique, essentiellement par augmentation pondérale de l'organe.

\section{Analyse de la grappe ovarienne}

Dans le tableau 3 figurent le poids, le contenu total en riboflavine et la concentration en cette vitamine d'une grappe ovarienne prélevée entièrement lors de chaque sacrifice (valeurs moyennes).

TABLEAU 3

Caractéristiques moyennes (poids et richesse en ribsfavine) d'une grappe ovarienne.

\begin{tabular}{|c|c|c|c|c|c|c|}
\hline \multirow[b]{2}{*}{$\begin{array}{l}\text { Date du } \\
\text { sacrifice }\end{array}$} & \multicolumn{3}{|c|}{ Lot témoin (régime S) } & \multicolumn{3}{|c|}{ Lot carencé (régime C) } \\
\hline & Poids (g) & $\begin{array}{l}\text { Contenu total } \\
\text { en riboflavine } \\
(\mu g)\end{array}$ & $\begin{array}{l}\text { Teneur en } \\
\text { riboflavine } \\
\text { ( } \text { gg/g frais) }\end{array}$ & Poids $(\mathrm{g})$ & $\begin{array}{c}\text { Contenu total } \\
\text { en riboflavine } \\
(\mu g)\end{array}$ & $\begin{array}{c}\text { Teneur en } \\
\text { riboflavine } \\
\text { ( } \mu \mathrm{g} / \mathrm{g} \text { frais) }\end{array}$ \\
\hline 6 avril ..... & 11,2 & 16,0 & $1, x^{\prime}$ & & & \\
\hline 17 avril ..... & 28,1 & 68,3 & 2,5 & 27,0 & 24,9 & 0,9 \\
\hline 27 avril ..... & 40,3 & 97,2 & 2,5 & 29,4 & $14, t^{\prime}$ & 0,5 \\
\hline 8 mai... & 35,5 & 78,2 & 2,1 & 8,8 & 5,7 & 0,8 \\
\hline \multirow[t]{2}{*}{15 mai........ } & 35,8 & 86,5 & 2,4 & - & 一 & - \\
\hline & \multicolumn{3}{|c|}{ Régime C à partir du 10 mai } & \multicolumn{3}{|c|}{$\begin{array}{l}\text { Administration per os d'une dose } \\
\text { unique de riboflavine, le } 10 \text { mai } \\
\text { (10 mg par poule) }\end{array}$} \\
\hline 15 mai... & 27,0 & 44,8 & 1,6 & 7,7 & 10,8 & 1,4 \\
\hline
\end{tabular}

Le poids de la grappe ovarienne est un reflet fidèle de l'intensité de ponte. La carence en riboflavine l'abaisse d'autant plus que les effets de l'avitaminose se font sentir davantage.

La carence affecte à la fois la concentration en riboflavine des grappes et leur contenu total en cette vitamine. $\mathrm{L}_{\mathbf{a}}$ teneur en vitamine $\mathrm{B}_{2}$ est fonction à la fois de 
la richesse des ovocytes et de celle du tissu ovarien. Or, la teneur du tissu ovarien est plus constante que celle des ovocytes. Dans un travail précédent (BLUM, CALET, JACQUOT, I96I), nous avons montré qu'avec un régime équilibré la teneur de la grappe variait avec l'intensité de ponte : lorsque celle-ci se ralentit, cette teneur tombe à $I \cdot I, 5 \mu g$ de riboflavine par gramme frais ce qui correspond à une prédominance du tissu ovarien. C'est ce qu'on observe pour la grappe des poules témoins sacrifiées le 6 avril alors que leur indice de ponte était très faible. La carence en riboflavine ne fait qu'accentuer le phénomène, car elle se manifeste intensément au niveau de la grappe ovarienne.

Après ro jours de régime $\mathrm{C}$, alors que les réserves hépatiques en riboflavine ne sont diminuées que de $30 \mathrm{p}$. Ioo par rapport aux témoins, le contenu de la grappe ovarienne est diminué de $70 \mathrm{p}$. Ioo. La disproportion est encore plus marquée pour la teneur en cette vitamine par gramme d'organe, soit -20 p. Ioo dans le foie et -65 p. roo dans la grappe ovarienne. Enfin la ponte s'arrête alors que le foie a encore près de la moitié de son capital en riboflavine.

L'administration per os d'une dose unique de riboflavine (Io mg par poule) 5 jours avant le sacrifice ne s'accompagne pas d'une reprise de l'activité ovarienne. Ce fait ne doit pas surprendre, car le développement de la grappe ovarienne, et partant la ponte, ne sont qu'indirectement sous la dépendance du régime, le contrôle direct revenant aux glandes endocrines, et d'abord à l'hypophyse. C'est ainsi que HosodA et al. (I955 et I956) puis MorRis et NALBANDOv (I96r) ont montré que la ponte, arrêtée par le jeûne total, peut être prolongée quelques jours en injectant des gonadotrophines hypophysaires. Si les Io $\mathrm{mg}$ de ribollavine ont permis à la poule de reconstituer son capital hépatique, il lui faudra néanmoins un certain temps pour que son fonctionnement hormonal se normalise, entrainant ainsi une reprise de l'activité ovarienne.

\section{Analyse du vitellus de l'cui}

Dans le tableau 4 figure pour chaque période considérée, la moyenne des teneurs en riboflavine du vitellus.

\section{TABLEAU 4}

Teneurs moyennes du vitellus cn ribofacine ( $\mu g / g$ frais)

\begin{tabular}{c|c|c|c|c|c|c}
\hline Dates & $\begin{array}{c}15 \text { au 25 } \\
\text { mars }\end{array}$ & $\begin{array}{c}26 \text { mars au } \\
5 \text { avril }\end{array}$ & $\begin{array}{c}6 \text { au 16 } \\
\text { avril }\end{array}$ & $\begin{array}{c}17 \text { au } 26 \\
\text { avril }\end{array}$ & $\begin{array}{c}27 \text { avril } \\
\text { au } 7 \text { mai }\end{array}$ & $\begin{array}{c}8 \text { au } 15 \\
\text { mai }\end{array}$ \\
\hline $\begin{array}{c}\text { Lot témoin } \\
\text { (régime S) }\end{array}$ & 3,3 & 2,6 & 3,0 & 3,0 & 2,9 \\
\hline $\begin{array}{c}\text { Lot carencé } \\
\text { (régime C) }\end{array}$ & 3,0 & 2,7 & 1,1 & 0,7 & 0,6 & - \\
\hline
\end{tabular}

Ce mode de présentation a l'inconvénient de ne pas tenir compte de l'aptitude propre de chaque pondeuse pour le transfert de la riboflavine à l'œuf. Cet inconvénient se fait surtout sentir pour les dernières périodes qui n'intéressent plus qu'un nombre réduit d'animaux, les autres ayant été préalablement sacrifiés. Pour pallier ce défaut, nous avons affecté, pour chaque poule: la moyenne des teneurs en ribo- 
flavine pendant la première période ( 15 au 25 mars) du coefficient Ioo. La teneur du vitellus des œufs suivants s'exprime alors en pourcentage de la première période. C'est la moyenne de ces pourcentages qui figure dans le tableau 5 .

TABLEAU 5

Évolution de la teneur en riboflavine du vitellus à partir de la première période considérée comme égale à Ioo

\begin{tabular}{|c|c|c|c|c|c|c|}
\hline Dates & $\begin{array}{l}15 \text { au } 25 \\
\text { mars }\end{array}$ & $\begin{array}{l}26 \text { mars aul } \\
5 \text { avril }\end{array}$ & $\begin{array}{c}6 \text { au } 16 \\
\text { avril }\end{array}$ & $\begin{array}{l}17 \text { au } 26 \\
\text { avril }\end{array}$ & $\begin{array}{l}27 \text { avril } \\
\text { au } 7 \text { mai }\end{array}$ & 8 au 15 \\
\hline $\begin{array}{l}\text { Lot témoin } \\
\text { (régime S) }\end{array}$ & 100 & $8:$ & 72 & 96 & 102 & 103 \\
\hline $\begin{array}{l}\text { Lot carencé } \\
\text { (Régime C) }\end{array}$ & 100 & 86 & $3 t$ & 25 & 12 & - \\
\hline & \multicolumn{6}{|c|}{ Intervention de la carence. } \\
\hline
\end{tabular}

De ces deux tableaux, il ressort que :

- La carence a un effet rapide et très marqué, entraînant en ro jours une chute de la teneur du vitellus d'environ 70 p. Ioo, alors qu'à cette époque la concentration de riboflavine dans le foie n'était guère diminuée que de ro p. Ioo et le capital hépatique de $30 \mathrm{p}$. Ioo.

- Chez les témoins, la teneur en riboflavine du vitellus diminue tout d'abord pour atteindre un minimum (-30 p. Ioo) situé entre le 6 et le $\mathrm{I} 6$ avril et qui coïncide avec l'indice de ponte le plus faible. La teneur du jaune d'œuf redevient normale lors de la reprise de ponte. Cette diminution passagère de la riboflavine du vitellus est à rapprocher de celle qui survient en fin de saison de ponte, alors que la production des œufs diminue peu de temps avant l'arrêt total précédant la mue (BLuM CALET, JaCguOT, I96I).

\section{Analyse de l'albumen de l'auf}

Ia moyenne des teneurs en riboflavine de l'albumen est indiquée dans le tableau 6 pour chaque période considérée. Dans le tableau 7 nous utilisons le même artifice que pour le vitellus en affectant la première période du coefficient roo.

TABLEAU 6

Teneur moyenne de l'albumen en riboflavine ( $\mu \mathrm{g} / \mathrm{g}$ frais)

\begin{tabular}{c|c|c|c|c|c|c}
\hline Dates & $\begin{array}{c}15 \text { au 25 } \\
\text { mars }\end{array}$ & $\begin{array}{c}26 \text { mars au } \\
5 \text { avril }\end{array}$ & $\begin{array}{c}6 \text { au 16 } \\
\text { avril }\end{array}$ & $\begin{array}{c}17 \text { au 26 } \\
\text { avril }\end{array}$ & $\begin{array}{c}27 \text { avril } \\
\text { au } 7 \text { mai }\end{array}$ & $\begin{array}{c}8 \text { au 15 } \\
\text { mai }\end{array}$ \\
\hline $\begin{array}{c}\text { Lot témoin } \\
\text { (régime S) }\end{array}$ & 2,5 & 2,2 & 2,6 & 2,6 & 2,6 & 2,5 \\
\hline $\begin{array}{c}\text { Lot carencé } \\
\text { (régime C) }\end{array}$ & 2,5 & 2,3 & 1,5 & 0,6 & 0,2 & - \\
\hline
\end{tabular}


TABLEAU 7

Évolution de la teneur en riboflavine de l'albumen à partir de la première période considérée comme égale à Ioo

\begin{tabular}{c|c|c|c|c|c|c}
\hline Dates & $\begin{array}{c}15 \text { au 25 } \\
\text { mars }\end{array}$ & $\begin{array}{c}26 \text { mars au } \\
5 \text { avril }\end{array}$ & $\begin{array}{c}6 \text { au 16 } \\
\text { avril }\end{array}$ & $\begin{array}{c}17 \text { au 26 } \\
\text { avril }\end{array}$ & $\begin{array}{c}27 \text { avril } \\
\text { au } 7 \text { mai }\end{array}$ & $\begin{array}{c}8 \text { au } 15 \\
\text { mai }\end{array}$ \\
\hline $\begin{array}{c}\text { Lot témoin } \\
\text { (régime S) }\end{array}$ & 100 & 87 & 97 & 100 & 112 & 115 \\
\hline $\begin{array}{c}\text { Lot carencé } \\
\text { (régime C) }\end{array}$ & 100 & 92 & 52 & 22 & 14 \\
\hline
\end{tabular}

Là encore l'influence de la carence se fait sentir nettement et avec une intensité plus grande qu'au niveau du tissu hépatique surtout dans les dernières périodes avant 1'arrêt total de la ponte. Chez les témoins la diminution transitoire de la teneur en riboflavine est moins marquée et plus précoce au niveau de l'albumen que dans le vitellus. Cette précocité, il est vrai, peut être due au fait que la formation du blanc d'œuf est plus rapide que celle du jaune.

\section{Conclusions}

En cas d'insuffisance d'apport en riboflavine, la poule peut sacrifier plus de $5^{\circ} \mathrm{p}$. Ioo de son capital hépatique en cette vitamine pour assurer la ponte, mais les derniers œufs pondus ont une teneur très faible en vitamine $B_{2}$.

Lorsque la carence survient en pleine ponte (Io mai), le capital hépatique est réduit de 40 p. Ioo en 5 jours sans que la teneur en vitamine $B_{2}$ des œufs pondus soit beaucoup diminuée. Ce qui permet de penser qu'une certaine proportion de la riboflavine $\mathrm{d} u$ foie constitue une réserve immédiatement mobilisable pour l'ovogenèse. Cette réserve serait à la fois fonction de l'état physiologique (hypertrophie du foie chez la pondeuse), de la richesse en riboflavine et du niveau protéique de la ration (quantité et qualité des protéines). Lorsque cette réserve est épuisée, le foie devient très avare de sa riboflavine et la teneur des oufs baisse considérablement, puis la ponte s'arrête.

A ce point de vue, la ponte n'est pas un phénomène prioritaire, l'organisme ne lui sacrifiant largement sa riboflavine que dans la mesure où ce sacrifice ne gêne pas ses fonctions vitales. Lors de la réplétion en vitamine $B_{2}$, les réserves sont d'abord reconstituées, l'activité ovarienne ne reprenant que plus tard.

\section{II. - Cinétique du dÉPôt de RIBOFLAVINE DANS L'AlBUMEN}

Nous avons montré que la riboflavine, comme les protéines (PEARL et CURTIS, I9I2), se déposait dans l'albumen au niveau du magnum (BLUM, CALET, JACQUOT, I96I). L'étude de la sécrétion de cet organe et de sa teneur en vitaine $B_{2}$ chez la poule normale ou carencée nous apporte des renseignements complémentaires. 


\section{Infuence de la carence sur l'albumen des outs pondus}

Dans notre essai, la carence en riboflavine était instituée à une époque où l'intensité de ponte allait en diminuant. Certaines poules ont alors continué à pondre. D'autres se sont arrêtées pendant un temps variable.

Dans le premier cas (maintien de ponte), la teneur en riboflavine de l'albumen diminuait progressivement à mesure que s'intensifiait la carence.

Dans la seconde éventualité, la ponte reprenait après un délai variant de 7 à 25 jours, selon deux types de modalités :

- l'albumen des œufs nouvellement pondus était très pauvre en riboflavine, ce qui indiquait que le magnum ne disposait plus d'aucune réserve faute d'un ravitail. lement suffisant ;

- à la reprise de ponte, l'albumen des deux premiers œufs avait une teneur élevée en riboflavine qui s'effondrait dans les œufs suivants. Ce fut le cas des poules 9 et I3 qui se remirent à pondre après un arrêt respectif de 9 et de 7 jours (tableau 8 ).

TABLEAU 8

Effet de la carence sur la teneur en riboflaine des oufs des poules 9 et i 3 .

\begin{tabular}{|c|c|c|c|c|}
\hline & & $\begin{array}{c}\text { Teneur des oufs } \\
\text { avant carence } \\
\text { ( } \mu \mathrm{g} / \mathrm{g} \text { frais) }\end{array}$ & $\begin{array}{c}\text { l'eneur des } 2 \text { premiers } \\
\text { cufs pondus après } \\
\text { intervention de la } \\
\text { carence } \\
(\mu \mathrm{g} / \mathrm{g} \text { frais })\end{array}$ & $\begin{array}{l}\text { Teneur des deux } \\
\text { cufs suivants } \\
(\mu \mathrm{g} / \mathrm{g} \text { frais })\end{array}$ \\
\hline Poule 9 & $\begin{array}{l}\text { Albumen } \ldots \ldots \\
\text { Vitellus ........ }\end{array}$ & $\begin{array}{l}2,1 \\
2,5\end{array}$ & $\begin{array}{l}2,0 \\
0,5\end{array}$ & $\begin{array}{l}0,3 \\
0,5\end{array}$ \\
\hline Poule 13 & $\begin{array}{l}\text { Albumen } \ldots \ldots \ldots \\
\text { Vitellus } \ldots \ldots \ldots \ldots\end{array}$ & $\begin{array}{l}2,{ }^{\prime} \\
2,9\end{array}$ & $\begin{array}{l}2,5 \\
1,1\end{array}$ & $\begin{array}{l}0,3 \\
0,6\end{array}$ \\
\hline
\end{tabular}

Nous avions malheureusement mélangé les albumens du premier et du second œuf pondus et nous ne pouvons savoir s'ils avaient les mêmes teneurs en riboflavine. Quoi qu'il en soit, l'organisme de ces poules semblait avoir épuisé une grande partie de la riboflavine disponible pour la ponte comme en témoigne la pauvreté du vitellus en cette vitamine. Il est d'autant plus surprenant de constater la richesse passagère de l'albumen. On peut penser avec BoLTON (I952) que toute la riboflavine nécessaire à l'albumen est accumulée dans le magnum entre chaque sécrétion. Il faut admettre la possibilité d'un mécanisme régulateur qui protégerait provisoirement cette réserve du magnum en dépit d'un état carentiel généralisé. C'est la seule hypothèse qui pourrait expliquer la richesse en riboflavine de l'albumen des deux premiers œufs nouvellement pondus qui fait contraste avec la pauvreté de l'albumen des œufs suivants. Mais il n'y a là qu'une possibilité puisque, dans la majorité des cas, la carence en riboflavine retentit immédiatement sur la teneur de l'albumen, qu'il y ait ou non arrêt de ponte.

\section{Étude du magnum et de sa sécrétion}

Afin de préciser la cinétique du dépôt de riboflavine dans le blanc d'œuf nous avons recueilli le magnum et l'albumen en voie de formation dans cet organe, chaque fois qu'un vitellus s'y trouvait engagé au moment du sacrifice. Le magnum était divisé en trois fractions : partie antérieure responsable de la sécrétion, partie contenant vitel- 
lus et albumen en voie de formation, partie postérieure n'ayant encore rien sécrété. A titre de comparaison, nous avons également prélevé l'isthme, organe histologiquement comparable au magnum. La riboflavine de ces différents prélèvements a été dosée et cela chez des Poules témoins recevant de la riboflavine (régime S) et chez des Poules carencées en cette vitamine (régime C). L,es valeurs des tableaux 9 (témoins) et Io (carencées) sont exprimées par centimètre d'organe et non par unité pondérale afin d'avoir une base de comparaison sans tenir compte de la sécrétion. Le poids du magnum et de l'isthme a été calculé par unité de longueur afin de rendre compte de l'activité sécrétoire de chaque segment. Ces tableaux mentionnent également le contenu en riboflavine de l'albumen en voie de formation et du blanc des derniers œufs pondus.

En dehors de toute déficience d'apport (témoins au régime S) le magnum de la poule pondeuse est un organe riche en riboflavine : son contenu total en vitamine $B_{2}$ aux stades I, III et IV est en moyenne de I69 $\mu \mathrm{g}$ pour un poids moyen de $20-25 \mathrm{~g}$. Le contenu hépatique total est de l'ordre de $700 \mu \mathrm{g}$ pour un poids du foie de $60-70 \mathrm{~g}$. La concentration en vitamine $\mathrm{B}_{2}$ est sensiblement de même ordre dans les deux organes. La teneur de l'isthme est nettement plus faible.

Chez ces poules témoins, aux stades pré- et postsécrétoires (I, III et IV) il existe un gradient de répartition de la riboflavine dans le magnum. Les teneurs les plus élevées par cm d'organe se trouvent dans la partie antérieure et les teneurs les plus faibles dans la partie postérieure, la partie moyenne ayant un taux intermédiaire. Au stade II, lorsque le vitellus est engagé dans le magnum, la chute des teneurs en riboflavine de la partie antérieure du magnum des poules témoins 4 et I 2 (tableau 9 ) correspond au transfert de cette vitamine dans l'albumen en voie de formation. Il est possible, cependant, que les réstultats concernant l'albumen pèchent par défaut du fait de la difficulté de récolter in situ cette production.

La richesse du magnum en riboflavine est variable d'une poule à l'autre à l'intérieur du lot témoin (tableau 9). Elle n'a qu'un rapport assez vague avec la richesse moyenne de 1'albumen des cufs pondus. Il semble que la recharge en vitamine du magnum ne soit pas toujours complète après passage du vitellus, ce qui expliquerait l'irrégularité des teneurs en riboflavine du blanc des œufs d'une même poule maintenue au même régime (ADRIAN, I958).

I a déficience d'apport de riboflavine abaisse considérablement la teneur du magnum en cette vitamine : le contenu total de $\mathrm{x} 69 \mu \mathrm{g}$ en moyenne chez les témoins, tombe à 22-23 $\mu \mathrm{g}$ après forte carence (poules 9 et 34 ). Dans ces conditions, on n'observe plus de gradient de répartition : la partie antérieure du magnum devient aussi pauvre que la partie postérieure. L'égalisation, qui se fait essentiellement aux dépens de la partie antérieure, précède l'épuisement général du magnum et l'effondrement des teneurs de l'albumen en voie de formation. Une courte carence de 5 jours conduit déjà à une baisse significative du taux de riboflavine dans la partie antérieure, alors que la partie postérieure est moins touchée (poules 3 et 32 ).

D'une manière générale, la richesse de l'albumen en riboflavine est sous le double contrôle de la place occupée dans le magnum et de la teneur de cet organe en vitamine $B_{2}$. Elle ne dépend pas du poids frais d'albumen sécrété. Ainsi dans le lot témoin (tableau 9), le poids d'albumen en voie de formation chez la poule 4 était de $3 \mathrm{~g}$ avec un contenu total de 5 I $\mu$ g de riboflavine alors que chez la poule I 2 le poids sécrété était de $6, \mathrm{I} 7 \mathrm{~g}$ avec un contenu total de $3 \mathrm{I}, 2 \mu \mathrm{g}$ de riboflavine. 


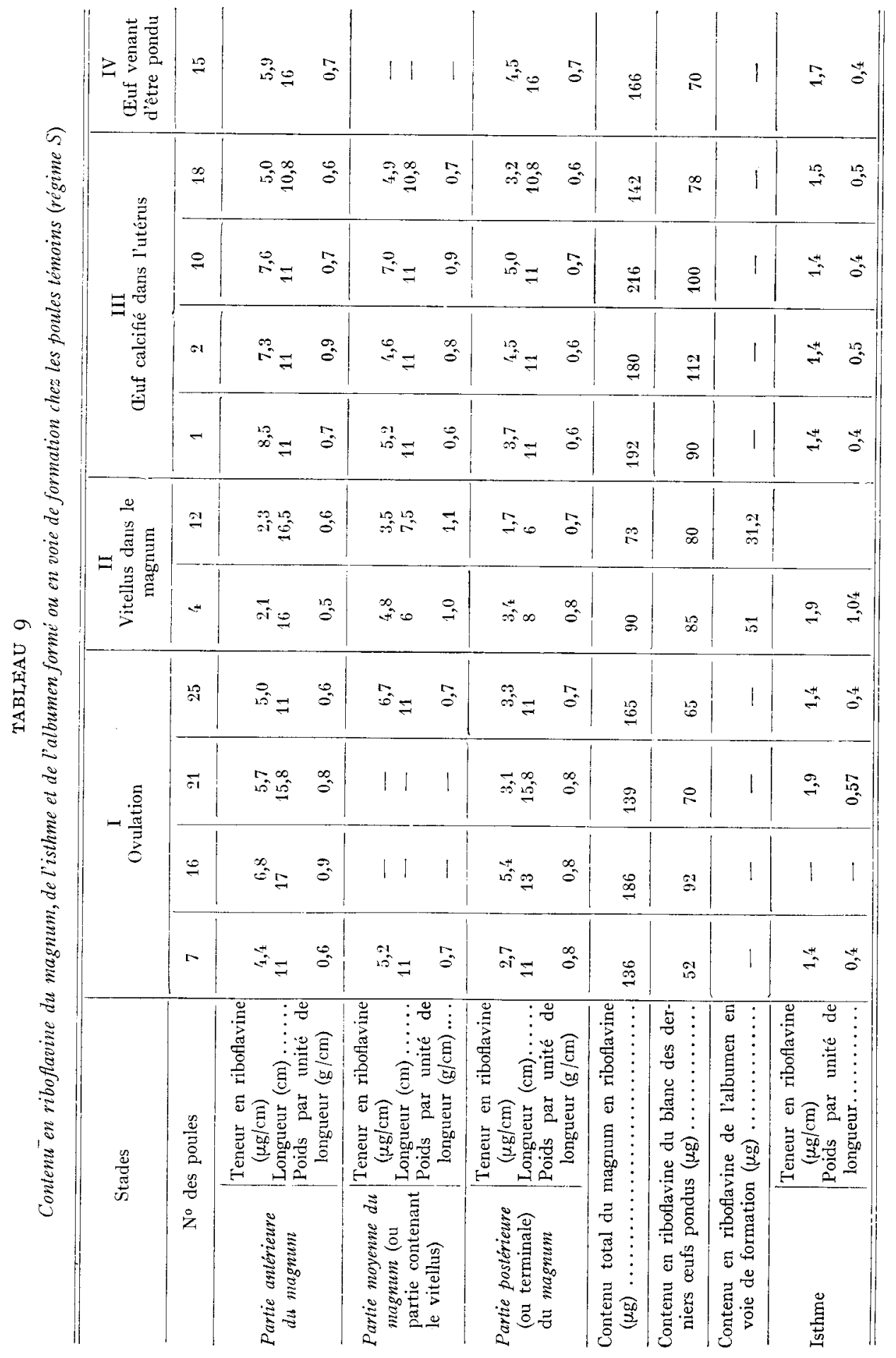




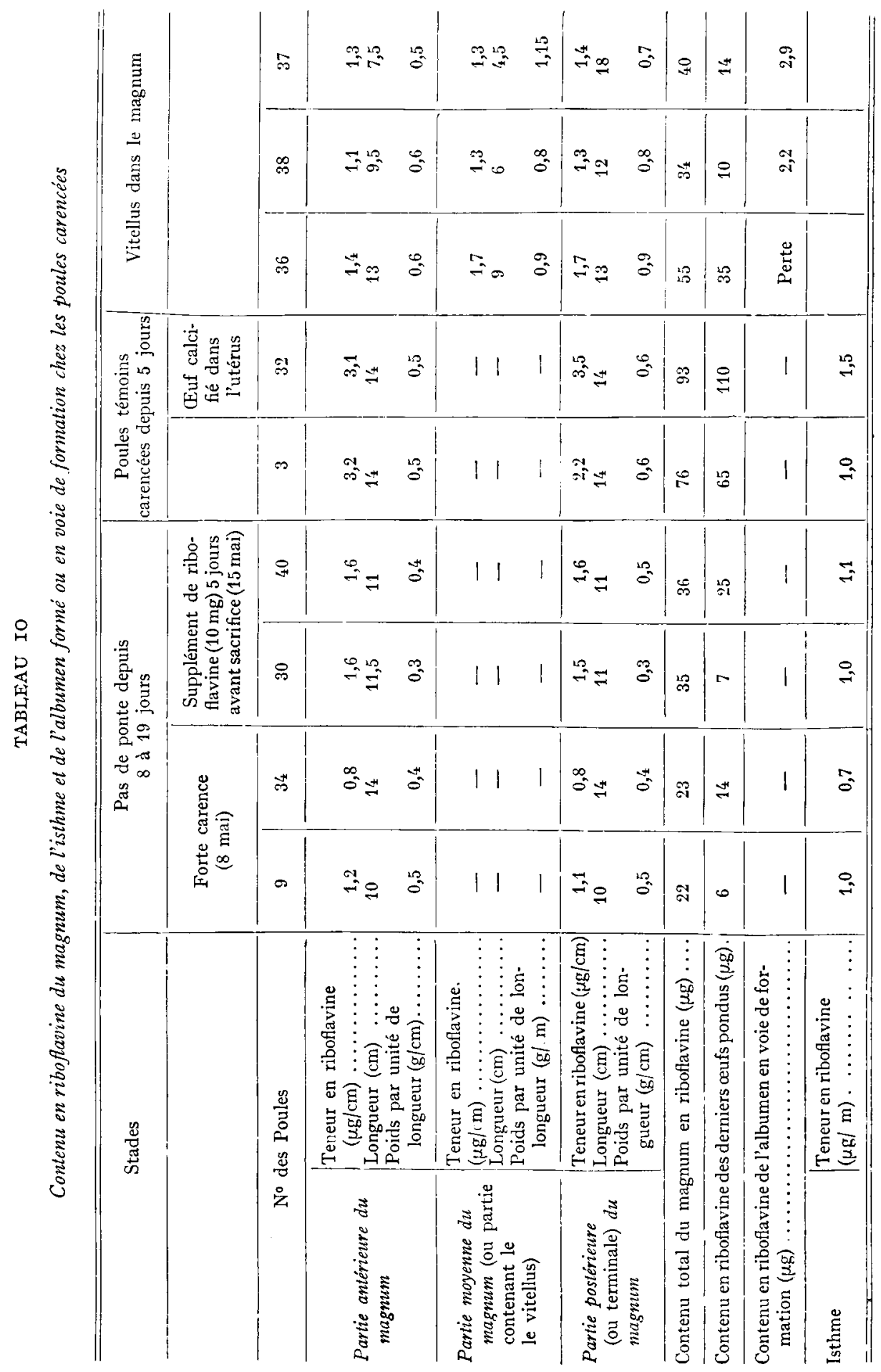

Annales de Biologie animale. - I062. 
Si l'on compare le contenu total en riboflavine du magnum et le contenu du blanc des derniers œufs pondus, on voit qu'en règle générale les réserves disponibles du magnum excèdent largement les quantités nécessaires à la formation de l'albumen et cela aussi bien chez les témoins (tableau 9) que chez les poules carencées (tableau Io) quels que soient les écarts notables des teneurs en valeur absolue.

\section{Conchusions}

Toute la riboflavine déposée dans l'albumen se trouve dans le magnum avant passage du vitellus.

Avec un régime contenant de la riboflavine, le magnum constitue des réserves de vitamine $B_{2}$ qui sont ensuite transférées à l'albumen, les deux tiers antérieurs jouant un rôle privilégié à cet égard. En effet, les réserves en riboflavine du magnum sont réparties selon un gradient de densité qui va en diminuant de la partie antérieure à la partie postérieure. La richesse en riboflavine dı magnum varie d'une poule à l'autre et n'a pas de rapports évidents avec la richesse du blanc des œufs pondus. Cette dernière est également indépendante de la quantité d'albumen secrété.

En régime carencé en riboflavine. le magnum ne se recharge pas normalement en vitamine $B_{2}$ et on n'observe plus de gradient de répartition entre les parties antérieure et postérieure. Dans ces conditions, deux types de transfert à l'albumen sont possibles. Lorsque la ponte se maintient, la carence retentit directement sur la teneur en riboflavine dı blanc d'œuf qui s'effondre. Lorsque la ponte s'arrête temporairement, la carence ne retentit pas nécessairement lors de la reprise de ponte sur le blanc des deux premiers oufs pondus qui renferme une teneur élevée de riboflavine, ce qui indique que l'albumen a pu sauvegarder partiellement ses réserves pendant l'arrêt de ponte. Mais, dès le $3^{\mathrm{e}}$ œuf, le blanc a un taux vitaminique très bas.

\section{CONCLUSIONS GÉNÉRALES}

La privation de vitamine $B_{2}$ imposée à la poule pondeuse conduit finalement à un arrêt de ponte qui est précédé par une réduction importante de la masse hépatique et du poids des grappes ovariennes allant de pair avec l'effondrement des teneurs en riboflavine du vitellus et de l'albumen.

Une partie seulement des réserves hépatiques de ribollavine est disponible pour l'ovogenèse. La carence retentit beaucoup plus sur le contenu en riboflavine des ovaires et de l'œuf que sur le contenu du foie. L'arrêt de ponte intervient alors que le foie possède encore la moitié environ de son capital en riboflavine. C'est dire que le besoin vitaminique de la ponte n'est pas prioritaire : la poule ne lui sacrifie ses réserves tissulaires que dans la mesure où ce transfert ne nuit pas à son propre équilibre nutritionnel. Ce comportement est à rapprocher des faits observés dans la gestation des Mam. mifères : la majorité des déficiences vitaminiques atteignent en premier lieu le fœtus sans qu'il soit le plus souvent possible de déceler le moindre signe clinique d'avitaminose chez la mère (JAcQuot, I953 ; BOURdel, JACQUOT, KLEIN et MAYER, I96I). $\mathrm{I}_{1}$ a priorité de l'organisme maternel ressort égalemeni du fait qu'une simple dose de Io $\mathrm{mg}$ de riboflavine administrée per os à la poule carencée suffit à reconstituer les réserves hépatian11es mais est incapable de provoquer la reprise de l'activité ovarienne. 
Chez la poule pondeuse non carencée, toute la ribollavine du blanc d'œuf provient des réserves du magnum, organe dont la teneur relative en vitamine $B_{2}$ n'est guère inférieure à celle du foie. Ces réserves sont localisées selon un gradient de concentration et leur importance diminue quand on passe de la partie antérieure à la partie postérieure du magnum. Dans l'albumen en voie de formation, prélevé in situ, il n'y a pas de relations évidentes entre le taux de transfert (contenu total en riboflavine) et la vitesse de sécrétion (poids d'albumen). La richesse vitaminique de l'albumen en voie de formation est à la fois fonction de la place occupée dans le magnum et de l'importance des réserves de cet organe. Il arrive que celui-ci n'arrive pas à se " recharger " pleinement entre le passage de deux vitellus, ce qui expliquerait les grandes variations du contenu en riboflavine du blanc des cufs de la même poule maintenue au même régime.

Chez la Poule carencée en vitamine $B_{2}$, on note une chute progressive des teneurs en riboflavine du magnum et de l'albumen. Comme quelques témoins, certaines poules carencées suspendent provisoirement leur ponte et, à la reprise, les deux premiers aufs nouvellement pondus peuvent avoir un albumen riche en riboflavine alors que le vitellus en est très pauvre. Le magnum parvient à sauvegarder une partie de ses réserves alors que la carence se fait déjà fortement sentir sur l'ovaire. Mais, ce n'est qu'un état passager et le blanc des oufs suivants devient très pauvre en riboflavine avant un arrêt définitif de ponte. Nos résultats montrent clairement que les modalités de transfert de la vitamine $B_{2}$ varient selon qu'il s'agit du vitellus ou de l'albumen. Les ovocytes et le vitellus sont normalement plus riches en riboflavine que le tissu ovarien alors que l'albumen est toujours plus pauvre que le magnum.

\section{Reçuen mai 1962.}

\section{SUMMARY}

THE EFFECTS OF SEVERE RIBOFLAVIN DEFICIENCY ON THE LAYING IIEN : THE ORDER OF PRIORITY BETWEEN THE MATERNAL TISSUES AND TIE EGG

Two batches of 20 layings hens were placed on a semi-synthetic diet (I 5 p. Ioo of protein in the form of gluten), the control group receiving to $\mathrm{mg}$ of riboflavin per $\mathrm{kg}$, the deficient group $0,4 \mathrm{mg}$ per $\mathrm{kg}$.

I. Distribution of riboflavin between the liver, the ovarian cluster and the egg.

The deficiency symptoms were as follows :

a) Egg laying fell off in all the animals before stopping entirely, on the 24 th day of deficiency.

b) The liver diminished considerably in volume, although the riboflavin content per gram of fresh liver tissue showed a smaller variation: on the 3 2nd day of deficiency the average liver weight of the deficient animals was 32 grams as compared with 70 grams in the control - a difference of 54 p. 100 ; the riboflavin content of the livers of the deficient batch was $8 \mu \mathrm{g}$ per gram of fresh tissue as compared with $1 x \mu \mathrm{g}$ in the control batch - a difference of $27 \mathrm{p} .100$

c) The ovarian cluster reacted more dramatically to the deficiency than the liver : on the $3^{2 n d}$ day its average weight was 9 grams in the deficient group and 36 grams in the control - a difference of 75 p. roo. The riboflavin content showed similar variation being. $0.8 \mu \mathrm{g}$ per gram of fresh tissue in the deficient batc has compared with $2.4 \mu \mathrm{g}$ per gram in the control - a difference of $67 \mathrm{p}$. I00. Thus while the vitamin deficiency had only a moderate effect upon the liver riboflavin content, it provoked a heavy drop in the amount of $\mathbf{B} 2$ present in the ovary.

d) The egg yolk showed a great sensibility to the deficiency ; in to days its riboflavin content had dropped $70 \mathrm{p}$. 10o, while the liver reserves had hardly been affected over this period. From this point the fall continued until on the $3^{2 n d}$ day : the riboflavin content was $0.6 \mu \mathrm{g}$ per gram of fresh yolk against $3 \mu \mathrm{g}$ per gram in the control- a difference of $80 \mathrm{p} .100$. 
e) The egg albumen is likewise more susceptible to the deficiency than the liver, and particularly during the later stages. The riboflavin content of fresh albumen was $0.2 \mu \mathrm{g}$ per gram in the deficient batch as compared with $2.6 \mu \mathrm{g}$ per gram in the control - a difference of $92 \mathrm{p}$. 100 .

f) Thus under conditions of ariboflavinosis only about $50 \mathrm{p}$. 100 of the liver storage of $\mathrm{B}_{2}$ are mobilized for egg production. Once this contribution has been made, the liver holds on to its vitamin, the egg riboflavin drops considerably and laying finally ceases. In this line egg laying is a phenomenon of a secondary order of priority, the maternal organism releasing its $\mathrm{B}_{2}$ storage only in so far as the maintenance of its own nutritional equilibrium will permit it. Furthermore, the B2 deficient hen will use any available riboflavin supply to re-establish its liver storage before making any $\mathrm{B}_{2}$ contribution for ovarian activity.

\section{The kinetics of riboflavin deposition in the albumen.}

All the ribotlavin contained in the white of the egg is obtained from the reserves accumulated in the magnum before the passage of the yolk :

a) in the non-deficient laying hen these reserves vary gradually, their importance diminishing from about $7 \mu \mathrm{g}$ to $4 \mu \mathrm{g}$ per centimetre in passing from the anterior to the posterior part of the magnum ;

b) in the deficient hen the magnum does not replace its $\mathrm{B}_{2}$ content normally and the anteriorposterior riboflavin supply gradient is no more observed.

\section{RÉFÉRENCES BIBLIOGRAPHIQUES}

ADRIAN J., I958. Variabilité et déterminisme des teneurs de l'œuf de poule en certaines vitamines du groupe B. Arch. Sc. Physiol., 12, 1-3o.

Adrian J., Lunven P., Terroine T., ig6r. Influence de la valeur protéique de la ration sur le taux urinaire et hépatique de la riboflavine, de la niacine et de la vitamine $\mathrm{B}_{6}$. Arch. Sc. Physiol., 15, $321-343$.

Blum J.-C., Calet C., Jaçuor R., ig6r. Note préliminaire sur les facteurs conditionnant la teneur de l'œuf en riboflavine. Ann. Biol. anim. Bioch. Biophys., 1, 31 I-316.

BoLTON W., 1952. Observations ont the vitamin metabolism of the common fowl. I. The partition of free and combined riboflavin in the tissues of the laying hen. Brit. J. Nutr., 6, $7 \mathrm{I} \cdot 75$.

Bourdel G., Jacquot R., Klein M., Mayer G., I96r. Nutrition et gestation. Ann. Nutr. Alim.. 15 $337-383$.

Fisher H., JoInsSON J. R., 1956. The aminoacid requirement of a free aminoacid diet for maintenance of egg production. I. The development of a free aminoacid diet for maintenance of egg production. $J$. Nutr. $60,26 \mathrm{I}-273$.

Hosoda T., Kaneko T., Mogi K., ABE T., I955. Effect of gonadotropic hormone on ovarian follicles and serum vittellin of fasting hens. Proc. Soc. Exper. Biol. Med., 88, 502-504.

Hosoda T., Kaneko T., MOGi K., AвE T., 1956. Forced ovulation in gonadotrophin-treated fasting hens. Proc. Soc. Exper. Biol. Med., 92, 360-362.

Jacovor R., 1953. Compétition alimentaire entre la mère et l'enfant. Chronologie des besoins. Ann. Nutr. Alim., 7, 39-57.

Morris T. R., Nalbandov A. V., Ig6r. The induction of ovulation in starving pullets using mammalian and avian gonadotropins. Endocrinology, 68, 687.697.

Pearl R., Curtis M. R., I9I2. Studies on the physiology of reproduction in the domestic fowl. V. Data regarding the physiology of the oviduct. - The absolute and relative amount of nitrogen in the albumen of eggs in different stages of formation. J. Exp. Zool., 12, I I 7-I 22.

Sneu E. F., Strong F. M., I939. A microbiological assay for riboflavin. Ind. Eng. Chem. Anal. Ed., 11, $43^{6}$. 